Journal of Algebraic Combinatorics 3 (1994), 285-290

(C) 1994 Kluwer Academic Publishers, Boston. Manufactured in The Netherlands.

\title{
Basis-Transitive Matroids
}

\author{
ANNE DELANDTSHEER \\ Service de Mathématiques, Faculté des Sciences Appliquées, C.P.165 Université Libre de Bruxelles, Av. Fr. \\ Roosevelt, 50, B-1050 Brussels, Belgium \\ HUILING LI \\ Dept. of Applied Mathematics, Zhejiang University, Hangzhou, Zhejiang, 310027, People's Republic of China
}

Received April 6, 1993; Revised August 23, 1993

\begin{abstract}
We consider the problem of classifying all finite basis-transitive matroids and reduce it to the classification of the finite basis-transitive and point-primitive simple matroids (or geometric lattices, or dimensional linear spaces). Our main result shows how a basis- and point-transitive simple matroid is decomposed into a so-called supersum. In particular each block of imprimitivity bears the structure of two closely related simple matroids, and the set of blocks of imprimitivity bears the structure of a point- and basis-transitive matroid.
\end{abstract}

Keywords: matroid, geometric lattice, dimensional linear space, transitivity, automorphism group

\section{Introduction}

Our aim is to investigate finite matroids admitting a basis-transitive automorphism group, which is essentially equivalent to the investigation of finite geometric lattices or of finite DLS's (dimensional linear spaces) admitting a basis-transitive automorphism group. For the definitions of matroid, geometric lattice and DLS, we refer the reader to Welsh [9] or White [10], Birkhoff [1] and Buekenhout [2] or Delandtsheer [5] resp. In 1985 Kantor [6] classified the finite simple matroids whose automorphism group acts transitively on ordered bases and called attention to the analogous problem for unordered bases. In this paper a matroid $\mathbf{M}$ will be called basis-transitive if its automorphism group AutM acts transitively on the bases of $M$ (i.e. on the maximal independent sets in $M$ ). We may neglect the loops of $M$ because they play absolutely no role in this problem. On the other hand if a basis-transitive automorphism group $G$ of $M$ has $t$ orbits $\mathbb{O}_{1}, \ldots, \mathbb{O}_{t}$ on the point-set $\mathbb{P}$ of $\mathbb{M}$, then the restriction of $M$ to each $\mathbb{O}_{i}$ is a matroid $\mathrm{M}_{i}$ on which $G$ acts point- and basis-transitively, and the matroid $M$ is the direct sum $\oplus_{i=1, \ldots, t} \mathbf{M}_{i}$ of these matroids. This has been proved in $\mathrm{Li} \mathrm{[7]} \mathrm{(Theorem} \mathrm{5)} \mathrm{for} \mathrm{simple} \mathrm{matroids,} \mathrm{but} \mathrm{the} \mathrm{proof} \mathrm{works} \mathrm{without}$ change for any matroid. Hence our problem reduces to point- and basis-transitive matroids.

Our next goal is to reduce the problem to simple matroids (or DLS's). To that end, consider a matroid $\mathbf{M}$ and call $\mathbb{P}_{1}, \ldots, \mathbb{P}_{t}$ its classes of parallel points (i.e. the maximal cliques of pairwise dependent points). If $G \leq$ Aut $M$ acts transitively on the points and bases of $M$, then all these classes have the same size $\omega$ and there is a transitive subgroup $H$ of the permutation group $\operatorname{Sym} \omega$ on $\omega$ points such that for any $i$, the action on $\mathbb{P}_{i}$ of the stabilizer of $\mathbb{P}_{i}$ in $G$ is isomorphic to $H$. On the other hand, the structure induced by $\mathbb{M}$ on the set $\left\{\mathbb{P}_{1}, \ldots, \mathbb{P}_{t}\right\}$ is precisely that of the simple matroid $\mathbb{T}$ canonically associated with 
$\mathbb{M}$, and AutM acts on $\mathbb{T}$ as a point-and basis-transitive automorphism group $K$. Moreover $G$ is a subgroup of the wreath product $H$ wr $K$.

Hence the investigation of basis-transitive matroids reduces to that of point- and basistransitive simple matroids (or DLS's). Since we shall mainly handle simple matroids, we will from now on use the DLS terminology. Point- and basis-transitive DLS's have been classified in dimension 2 and 3 (i.e. rank 3 and 4) by Li [7]. Delandtsheer [3] provides a classification of the 2-transitive and basis-transitive DLS's with line size $\neq 2$, and decomposes any point- and basis-transitive DLS with some line of size $>2$ into isomorphic 2-transitive and basis-transitive DLS's by means of a "wreath product" involving some point- and basis-transitive matroid $\mathbb{M}$. This "wreath product" generalizes the notion of direct sum of isomorphic DLS's, but is in turn a very special case of the notion of supersum introduced in Delandtsheer [4] and recalled in Section 2 since it is crucial for the statement of our main result.

The present paper generalizes Theorem 1.2 of Delandtsheer [3] in decomposing any pointand basis-transitive DLS into a supersum $\oplus_{\mathbb{M}}\left(\mathbb{R}, \mathbb{R}^{\prime}\right)$ where $\mathbb{R}, \mathbb{R}^{\prime}$ are point-primitive and basis-transitive DLS's and $\mathbb{M}$ is a point- and basis-transitive matroid. All the above mentioned results (including Kantor's one) are either reduction theorems and have an elementary proof, or are classification theorems of low dimension or highly transitive basis-transitive DLS's and rely on the classification of 2-transitive permutation groups (and thereby on the classification of finite simple groups). By the present reduction theorem the classification of all finite basis-transitive matroids amounts to that of all point-primitive and basis-transitive DLS's. The point-primitivity hypothesis (although weaker than 2-transitivity) might lead to a final classification. Possible tools are the O'Nan-Scott theorem classifying the primitive permutation groups into five major types together with the classification of finite simple groups.

\section{Definitions and statement of the theorem}

Let us first recall that an $n$-DLS is precisely a simple matroid of rank $n+1(0 \leq n<+\infty)$. We shall call pre- $n$-DLS any matroid of rank $n+1(-1 \leq n<+\infty)$. The m-truncation of an $n$-DLS $\mathcal{S}$ (with $m \leq n$ ) is the $m$-DLS, denoted by $m-\mathcal{S}$, whose independent sets are the independent sets of size $\leq m-1$ of $\mathcal{S}$.

The flats (or varieties) of dimension $i$ will be called $i$-flats. The flats of dimension 0 or 1 (resp. of codimension 1 or 2) will preferably be called points or lines (resp. hyperplanes or colines). An $i$-flat is called thick if it has more than $i+1$ points. A $(d, d-1)$-DLS association is a pair $\left(\mathbb{R}, \mathbb{R}^{\prime}\right)$ where $\mathbb{R}$ is a $d$-DLS $(0 \leq d<+\infty)$ on some point-set $\mathbb{P}$ and $\mathbb{R}^{\prime}$ is a pre- $(d-1)$-DLS such that every basis of $\mathbb{R}^{\prime}$ is contained in some basis of $\mathbb{R}$, every basis of $\mathbb{R}$ contains a basis of $\mathbb{R}^{\prime}$ and the hyperplanes of $\mathbb{R}^{\prime}$ are certain colines or hyperplanes of $\mathbb{R}$. The simplest possibility is that $\mathbb{R}^{\prime}$ is the $(d-1)$-truncation of $\mathbb{R}$, which means that the bases of $\mathbb{R}^{\prime}$ are precisely the independent $d$-sets of $\mathbb{R}$ (in other words, the hyperplanes of $\mathbb{R}^{\prime}$ are the colines of $\mathbb{R}$ ). There are however other possibilities: for example the hyperplanes of $\mathbb{R}^{\prime}$ might be a selection of hyperplanes of $\mathbb{R}$ together with the colines of $\mathbb{R}$ which are in none of these selected hyperplanes. 
Let $\mathbb{T}$ be a pre- $d_{0}$-DLS on the point-set $\{1, \ldots, t\}$, let $\mathbb{P}_{1}, \ldots, \mathbb{P}_{t}$ be pairwise disjoint sets and, for each $i=1, \ldots, t$, let $\left(\mathbb{R}_{i}, \mathbb{R}_{i}^{\prime}\right)$ be a $\left(d_{i}, d_{i}-1\right)$-DLS association on the pointset $\mathbb{P}_{i}$. If moreover the pair $\{i, j\}$ is independent in $\mathbb{T}$ whenever $d_{i}=d_{j}=0$, then the supersum of the $\left(\mathbb{R}_{i}, \mathbb{R}_{i}^{\prime}\right)$ 's over $\mathbb{T}$ is the $\left(\sum_{i=0, \ldots, t} d_{i}\right)$-DLS $\oplus_{\mathbf{T}}\left(\mathbb{R}_{i}, \mathbb{R}_{i}^{\prime}\right)$ with point-set $\cup_{i=1, \ldots, t} \mathbb{P}_{i}$ and whose bases are the sets $B$ for which there is a basis $J$ of $\mathbb{T}$ such that $B \cap \mathbb{P}_{j}$ is a basis of $\mathbb{R}_{j}$ if $j \in J$ and $B \cap \mathbb{P}_{j}$ is a basis of $\mathbb{R}_{j}^{\prime}$ if $j \notin J$. Note that if $\mathbb{T}$ is a boolean matroid (i.e. if $\left.d_{0}=t-1\right)$, then the supersum $\oplus_{\mathbf{T}}\left(\mathbb{R}_{i}, \mathbb{R}_{i}^{\prime}\right)$ reduces to the direct sum $\oplus_{i=1, \ldots, t} \mathbb{R}_{i}$. Note also that any pre-DLS $M$ with classes of parallel points $\mathbb{P}_{1}, \ldots, \mathbb{P}_{t}$ can be seen as the supersum $\oplus \mathbf{T}\left(\mathbb{R}_{\boldsymbol{i}}, \mathbb{R}_{i}^{\prime}\right)$ where $\mathbb{T}$ is the DLS canonically associated with $M$ and $\mathbb{R}_{i}$ (resp. $\mathbb{R}_{i}^{\prime}$ ) is the 0-DLS (resp. pre-(-1)-DLS) on $\mathbb{P}_{i}$.

In this paper, we will only need the special case where all $\left(\mathbb{R}_{i}, \mathbb{R}_{i}^{\prime}\right)$-associations are isomorphic to a common $(d, d-1)$-DLS-association. If moreover $\mathbb{R}^{\prime}$ is a $(d-1)$-DLS, then the supersum $\oplus_{\mathbf{T}}\left(\mathbb{R}, \mathbb{R}^{\prime}\right)$ is the "wreath product" needed in Delandtsheer [3] and already introduced by Lim [8] who calls it "direct product".

We can now state our main result:

Theorem. Let $\mathcal{S}$ be a finite DLS and let $G \leq A u t S$ be point- and basis-transitive. Then $\mathcal{S}$ is a supersum $\oplus_{\mathcal{T}}\left(\mathcal{R}, \mathcal{R}^{\prime}\right)$ where $\mathcal{T}$ is a point- and basis-transitive pre- $d_{0}-D L S$ with $d_{0} \geq 0$ and $\left(\mathcal{R}, \mathcal{R}^{\prime}\right)$ is a $(d, d-1)$-DLS association sharing a common point-primitive and basis-transitive automorphism group.

\section{Proof of the theorem}

By abuse of language, we will always speak of $G$ and of its $G$-orbits, no matter $G$ is to be considered as a permutation group acting on points, on bases or on other objects. If $G$ is primitive on the points of $\mathcal{S}$, there is nothing to prove. Suppose now that $\Delta_{1}, \Delta_{2}, \ldots, \Delta_{t}$ form a complete set of imprimitivity blocks and that $G_{\Delta_{i}}$ is primitive on $\Delta_{i}$. Then $t \geq 2$, $\left|\Delta_{i}\right| \geq 2$ and $\operatorname{dim}\left\langle\Delta_{i}\right\rangle \geq 1$. Moreover this cardinality and this dimension are independent of $i \in I=\{1, \ldots, t\}$. Let $d=\operatorname{dim}\left\langle\Delta_{i}\right\rangle$.

Given any basis $B$ of $\mathcal{S}$, we define $B_{i}=B \cap \Delta_{i}$ and $\beta_{i}=\left|B_{i}\right|$, so that a $t$-tuple $\left(\beta_{i}\right)_{i \in I}$ is attached to $B$. If $B^{\prime}$ denotes some other basis, $B_{i}^{\prime}$ and $\beta_{i}^{\prime}$ are defined accordingly. Any $t$-tuple attached to some basis of $\mathcal{S}$ will be called a basis-t-tuple. Since $G$ acts transitively on the bases of $\mathcal{S}, G$ also acts transitively on the basis$t$-tuples.

The maximal cardinality of the independent sets contained in $\Delta_{i}$ is $d+1 \geq 2$. Since any independent set assuming this bound can be extended to a basis of $\mathcal{S}$, we get $\max \left\{\beta_{i} ; i \in\right.$ $I\}=d+1 \geq 2$. Let us define $m:=\min \left\{\beta_{i} ; i \in I\right\}$. Since $\left(\beta_{i}\right)_{i \in I}$ refers to a distinguished basis $B$, we may arrange the index set $I$ so that $d+1=\beta_{1} \geq \beta_{2} \geq \cdots \geq \beta_{t}=m$. Suppose that $m<d+1$. Since $\left.1+\operatorname{dim}\left\langle\Delta_{t}\right\rangle=d+1\right\rangle m=\beta_{t}$, there is a point $y \in \Delta_{t}$ such that $B_{t} \cup y$ is independent. Thus $B^{\prime}=(B \backslash x) \cup y$ is a basis for some $x \in \Delta_{i} \cap B, i<t$. Hence $\beta_{t}^{\prime}=m+1$, so that $\beta_{j}=m+1$ for some $j<t$. Now define $u$ and $v$ as follows 


$$
\beta_{i}\left\{\begin{array}{lll}
\geq m+2 & \text { iff } & i \leq u \\
=m+1 & \text { iff } & u+1 \leq i \leq v \\
=m & \text { iff } & v+1 \leq i \leq t
\end{array}\right.
$$

so that $0 \leq u<v<t$.

Step 0. $\Delta_{v+1} \cup \cdots \cup \Delta_{t} \subseteq\left\langle B_{u+1} \cup \cdots \cup B_{t}\right\rangle$

Proof: Let $\mathcal{A}$ be the set of all subsets $A$ of $B$ such that

$$
\left|A \cap \Delta_{i}\right|=\left\{\begin{array}{lll}
m+1 & \text { if } & 1 \leq i \leq v \\
m & \text { if } \quad v+1 \leq i \leq t
\end{array}\right.
$$

Clearly $A \cap \Delta_{i}=B_{i}$ for $u+1 \leq i \leq t$, and if $A \neq A^{\prime}$ are in $\mathcal{A}$, then $A \cap \Delta_{j} \neq A^{\prime} \cap \Delta_{j}$ for some $j \leq u$. Moreover $\cap_{A \in \mathcal{A}} A=B_{u+1} \cup \cdots \cup B_{t}$.

If there is a point $y$ such that $y \in \Delta_{v+1} \cup \cdots \cup \Delta_{t}$ and $y \notin\langle A\rangle$, then $A \cup y$ is independent and can be extended to a basis $B^{\prime}$. But for $B^{\prime}$ there are at least $v+1$ indices $j$ such that $\beta_{j}^{\prime} \geq m+1$, which contradicts the basis-transitivity assumption or the definition of $v$. Hence $\Delta_{v+1} \cup \cdots \cup \Delta_{t} \subseteq\langle A\rangle$. Consequently

$$
\Delta_{v+1} \cup \cdots \cup \Delta_{t} \subseteq \bigcap_{A \in \mathcal{A}}\langle A\rangle=\left\langle\bigcap_{A \in \mathcal{A}} A\right\rangle=\left\langle B_{u+1} \cup \cdots \cup B_{t}\right\rangle .
$$

Step 1. $m \geq 1$.

Suppose for a contradiction that $m=0$. Note that $u \geq 1$ since $m+1<d+1=2$. For $j \in\{u+1, \ldots, v\}$, pick a point $y_{j}$ in $\Delta_{j} \backslash B_{j}$. Then the independent set $B_{j} \cup y_{j}$ can be extended to a basis $B^{\prime}$ by adjoining points in $B$ only. Hence $B^{\prime}=(B-x) \cup y_{j}$ for some $x \notin B_{j}$, and $\beta_{j}^{\prime}=2$. By basis-transitivity, $\beta_{i}^{\prime}=1$ for some $i \leq u$. Then applying Step 0 to the basis $B^{\prime}$, we get

$$
\Delta_{v+1} \cup \cdots \cup \Delta_{t} \subseteq\left\langle B_{i}^{\prime} \cup C_{j}\right\rangle
$$

where $C_{j}=B_{u+1} \cup \cdots \cup B_{j-1} \cup B_{j+1} \cup \cdots \cup B_{v}$.

Since $\left\langle B_{i}^{\prime} \cup C_{j}\right\rangle \cap\left\langle B_{u+1} \cup \cdots \cup B_{v}\right\rangle=\left\langle C_{j}\right\rangle$, Step 0 implies that

$$
\Delta_{v+1} \cup \cdots \cup \Delta_{t} \subseteq\left\langle C_{j}\right\rangle .
$$

But this holds for any $j=u+1, \ldots, v$, so that

$$
\Delta_{v+1} \cup \cdots \cup \Delta_{t} \subseteq \bigcap_{j=u+1, \ldots, v}\left\langle C_{j}\right\rangle=\left\langle\bigcap_{j=u+1, \ldots, v} C_{j}\right\rangle=\phi
$$

a contradiction.

Step 2. Any union of imprimitivity blocks is a flat of $\mathcal{S}$. In particular each $\Delta_{i}$ is a flat of $\mathcal{S}$ and $\operatorname{dim} \Delta_{i}=d$. 
Let us first prove that, for any $i \in I, \cup_{j \neq i} \Delta_{j}$ is a flat of $\mathcal{S}$. Since $G$ acts transitively on the $t$ imprimitivity blocks, there is a basis $B$ having $\beta_{i}=m$ points in $\Delta_{i}$. Since $m \geq 1$ and since $G_{\Delta_{i}}$ acts transitively on the points of $\Delta_{i}$, this implies that for any $x \in \Delta_{i}$ there is a basis, say $B(x)$, containing $x$ and intersecting $\Delta_{i}$ in exactly $m$ points. By the minimality of $m$, the hyperplane $\langle B(x) \backslash x\rangle$ contains all other blocks $\Delta_{j} \neq \Delta_{i}$. Hence $\cup_{j \neq i} \Delta_{j}=\cap_{x \in \Delta_{i}}\langle B(x) \backslash x\rangle$ is an intersection of hyperplanes, and so is a flat of $\mathcal{S}$. By intersection, any union of blocks is a flat of $\mathcal{S}$. In particular every $\Delta_{i}$ is a flat of $\mathcal{S}$ and its dimension is $d=\operatorname{dim}\left\langle\Delta_{i}\right\rangle$. Let us denote by $\mathbb{D}_{i}$ the $d$-DLS $(d \geq 1)$ induced by $\mathcal{S}$ on $\Delta_{i}$.

Step 3. $G_{\Delta_{i}}$ acts basis-transitively on the $d$-DLS $\mathbb{D}_{i}$.

Let $B$ be a basis of $\mathcal{S}$ such that $\beta_{1}=d+1$, so that $B_{1}=B \cap \Delta_{1}$ is a basis of $\mathbb{D}_{1}$. If $B_{1}^{\prime}$ is some other basis of $\mathbb{D}_{1}$, then $B^{\prime}=\left(B \backslash B_{1}\right) \cup B_{1}^{\prime}$ is also a basis of $\mathcal{S}$. Since $B$ and $B^{\prime}$ are in the same $G$-orbit, the $G$-orbit of $B_{1}$ must contain the same number of $B_{i}$ 's and

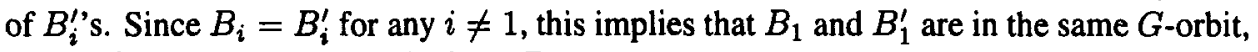
and so $G_{\Delta_{1}}$ acts basis-transitively on $\mathbb{D}_{1}$.

Step 4. $\left\{\beta_{i} ; i \in I\right\} \subseteq\{d, d+1\}$.

If $m=d+1$ or $d$, there is nothing to prove. Hence suppose that $m<d$. Then there are two points $y_{t}$ and $z_{t}$ in $\Delta_{t}$ such that $B_{t} \cup\left\{y_{t}, z_{t}\right\}$ is independent and can be extended to a basis $C$ of $\left\langle B_{u+1} \cup \cdots \cup B_{t}\right\rangle$. Thus $B^{\prime}=\left(B \backslash\left(B_{u+1} \cup \cdots \cup B_{t}\right)\right) \cup C$ is a basis of $\mathcal{S}$. But for $B^{\prime}$, there are at least $u+1$ indices $i \in I$ such that $\beta_{i}^{\prime}>m+1$, which is impossible.

Step 5. If $\left\{\beta_{i} ; i \in I\right\}=\{d+1\}$, then $\mathcal{S} \cong \oplus_{i \in I} \mathbb{D}_{i}$.

This is obvious, so we only have to investigate the case where $\left\{\beta_{i} ; i \in I\right\}=\{d, d+1\}$.

Step 6. The flats of $\mathcal{S}$ containing $\cup_{i \neq 1} \Delta_{i}$ provide $\Delta_{1}$ with the structure of a pre- $(d-1)$ DLS $\mathbb{D}_{1}^{\prime}$, so that the pair $\left(\mathbb{D}_{1}, \mathbb{D}_{1}^{\prime}\right)$ is a $(d, d-1)$-DLS association sharing a common point-primitive and basis-transitive automorphism group $G_{\Delta_{1}}$.

Let $B$ be a basis of $\mathcal{S}$ such that $\beta_{1}=d$. Arguing as in Step 3 shows that a set $B^{\prime}$ of the form $\left(B \backslash B_{1}\right) \cup B_{1}^{\prime}$ (where $\left.B_{1}^{\prime} \subset \Delta_{1}\right)$ is a basis of $\mathcal{S}$ if and only if $B_{1}^{\prime}$ belongs to the same $G_{\Delta_{1}}$-orbit $O_{1}$ as $B_{1}$. Moreover the elements of $O_{1}$ are the bases of the pre- $(d-1)$-DLS $\mathbb{D}_{1}^{\prime}$ defined on $\Delta_{1}$ by the closure operator

$$
A \subseteq \Delta_{1} \rightarrow \bar{A}=\Delta_{1} \cap\left\langle A \cup \Delta_{2} \cup \cdots \cup \Delta_{t}\right\rangle .
$$

Note that the empty set is closed in $\Delta_{1}$ since $U_{i \neq 1} \Delta_{i}$ is a flat of $\mathcal{S}$. On the other hand, if $d \geq 2$, then $\mathbb{D}_{1}^{\prime}$ is a $(d-1)$-DLS, because classes of parallel points would contradict our assumption that $G_{\Delta_{1}}$ acts primitively on $\Delta_{1}$.

Finally note that every basis of $\mathbb{D}_{1}^{\prime}$ is an independent set of $\mathcal{S}$, so that it can be extended to a basis of $\mathbb{D}_{1}$. Conversely the transitivity properties of $G$ imply that for every basis $B_{1}$ 
of $\mathbb{D}_{1}$, the generating set $B_{1} \cup\left(\Delta_{2} \cup \cdots \cup \Delta_{t}\right)$ contains a basis $B^{\prime}$ such that $B_{1}^{\prime} \in O_{1}$. Hence every basis $B_{1}$ of $\mathbb{D}_{1}$ contains a basis of $\mathbb{D}_{1}^{\prime}$. Given a basis-t-tuple $\left(\beta_{i}\right)$, define $J=\left\{i \in I ; \beta_{i}=d+1\right\}$. It is then immediate that

Step 7. The bases of $\mathcal{S}$ are precisely the sets $B$ such that $\left(\beta_{i}\right)$ is a basis-t-tuple and $B_{i}$ is a basis of $\mathbb{D}_{i}$ (resp. of $\mathbb{D}_{i}^{\prime}$ ) iff $i \in J$.

Now let $\mathbb{J}$ be the set of all $J$ 's associated to all bases of $\mathcal{S}$. It is easy to check that $\mathbb{J}$ is the set of bases of some pre-DLS $\mathbb{T}$ on $I$ and that $G$ acts point-and basis-transitively on $\mathbb{T}$. This ends the proof of the Theorem.

\section{References}

1. Birkhoff, G., Lattice Theory, Amer. Math. Soc. Colloq. Publ. (3rd ed.), Providence, 1967.

2. Buekenhout, F., "Diagrams for geometries and groups," J. Combin. Theory Ser. A 27 (1979), 121-151.

3. Delandtsheer, A., "Basis-homogeneous geometric lattices," J. London Math. Soc. (2) 34 (1986), 385-393.

4. Delandtsheer, A., Classifications of finite highly transitive dimensional linear spaces, Proc. Int. Conf. on Combinatorial Geom. 91, Capri, 1991, to appear in Discrete Math.

5. Delandtsheer, A., Dimensional linear spaces, to appear in the "Handbook of Incidence Geometry," F. Buekenhout ed., North Holland.

6. Kantor, W.M., "Homogeneous designs and geometric lattices," J. Combin. Theory Ser. A 38 (1985), 66-74.

7. Li, H., "On basis-transitive geometric lattices," European J. Combin. 10 (1989), 561-573.

8. Lim, M.H., "A product of matroids and its automorphism group," J. Combin. Theory Ser. B 23 (1977), 151-163.

9. Welsh, D.J.A., Matroid Theory, Academic Press, London, 1976.

10. White, N.L., Theory of matroids, Cambridge Univ. Press, Cambridge, 1986. 